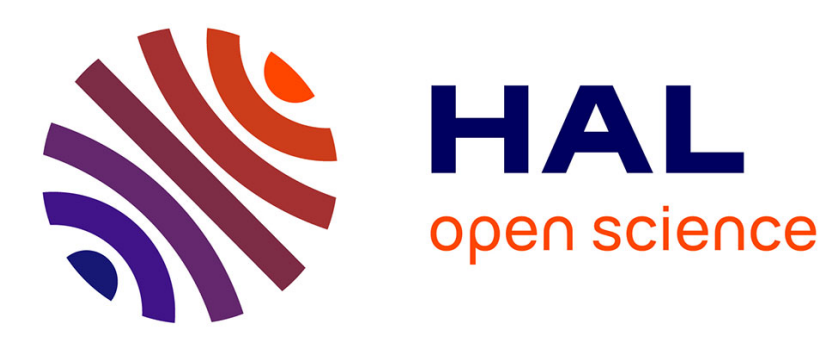

\title{
Des tuméfactions de la lèvre
}

Ludovic Hery, Thibault Fraisse, Franklin Samou, Anne-Sophie Zampaolo, Emilie Rigal, Carole Chevenet, Sébastien Trouillier

\section{To cite this version:}

Ludovic Hery, Thibault Fraisse, Franklin Samou, Anne-Sophie Zampaolo, Emilie Rigal, et al.. Des tuméfactions de la lèvre. La Revue de Médecine Interne, 2015, 36 (4), pp.294-5. 10.1016/j.revmed.2014.05.010 . hal-01152306

\section{HAL Id: hal-01152306 https://hal.science/hal-01152306}

Submitted on 15 May 2015

HAL is a multi-disciplinary open access archive for the deposit and dissemination of scientific research documents, whether they are published or not. The documents may come from teaching and research institutions in France or abroad, or from public or private research centers.
L'archive ouverte pluridisciplinaire HAL, est destinée au dépôt et à la diffusion de documents scientifiques de niveau recherche, publiés ou non, émanant des établissements d'enseignement et de recherche français ou étrangers, des laboratoires publics ou privés.

\section{(1)(1) $\$(0)$}

Distributed under a Creative Commons Attribution - NonCommercial - ShareAlikel 4.0 


\section{Des tuméfactions de la lèvre / Labial tumefaction}

L. Hery ${ }^{\mathrm{a},}{ }^{,}$,, T. Fraisse ${ }^{\mathrm{a}}$, F. Samou ${ }^{\mathrm{a}}$, A.-S. Zampaolo ${ }^{\mathrm{b}}$, E. Rigal $^{\mathrm{b}}$, C. Chevenet $^{\mathrm{c}}$, S. Trouillier $^{\mathrm{a}}$

a Service de médecine interne, centre hospitalier Henri-Mondor, 50, avenue de la République, 15000 Aurillac, France

b Service de dermatologie, centre hospitalier Henri-Mondor, 50, avenue de la République, 15000 Aurillac, France

${ }^{\mathrm{c}}$ Laboratoire d'anatomopathologie, 23, avenue de la République, 15000 Aurillac, France

${ }^{\mathrm{d}}$ Membre du consortium Couperin, pôle science de la vie et de la santé, maison des Universités, 103, boulevard Saint-Michel, 75005 Paris, France

* Auteur correspondant.

Mots clés : Amylose AL, Myélome multiple Artérite temporale

Keywords: AL amyloidosis, Multiple myeloma, Temporal arteritis

\section{L'histoire}

Un homme, âgé de 80 ans, consultait pour une dyspnée d'effort s'aggravant depuis 3 à 4 ans. Son médecin traitant signalait également une claudication de la mâchoire et des douleurs scapulaires inflammatoires depuis quelques semaines et évoquait le diagnostic d'une artérite à cellules géantes temporales. Ce patient avait un myélome à IgG Kappa stade III traité 10 ans auparavant par 12 cures de melphalan/prednisone et biphosphonates. Une leucémie lymphoïde chronique stade A était diagnostiquée un an après le myélome. Une vascularite nécrosante avec atteinte neurologique périphérique survenait 3 ans après le traitement du myélome et nécessitait un traitement par prednisone, 6 bolus de cyclophosphamide relayés par azathioprine pendant un an puis mycophénolate mofétil pendant 5 ans. Il avait également une cardiomyopathie ischémique pontée et valvulaire aortique.

À l'examen, les artères temporales étaient indurées et non battantes. On notait également une hypertrophie des deltoïdes, des quadriceps et muscles fessiers, une macroglossie, des tuméfactions de la face muqueuse de la lèvre inférieure (Fig. 1) ainsi qu'une ataxie proprioceptive invalidante. Il n'y avait pas d'insuffisance cardiaque ni d'anomalie auscultatoire pulmonaire. On notait une augmentation en 6 mois du pic de $16,1 \mathrm{~g} / \mathrm{L}$ à $19,1 \mathrm{~g} / \mathrm{L}$ sur l'électrophorèse des protéines, une protéinurie à $2,2 \mathrm{~g} / 24 \mathrm{~h}$, une insuffisance rénale (créatininémie : $130 \mathrm{moL} / \mathrm{L}$ ) et une anémie (taux d'hémoglobine : 10,5 g/dL), un syndrome inflammatoire (taux de fibrinogène : $6 \mathrm{~g} / \mathrm{L}, \mathrm{CRP}: 26 \mathrm{mg} / \mathrm{L}$ ). 


\section{Le diagnostic}

Une amylose AL avec atteinte musculaire, rénale, articulaire, temporale et salivaire compliquant un myélome multiple IgG Kappa.

\section{Les commentaires}

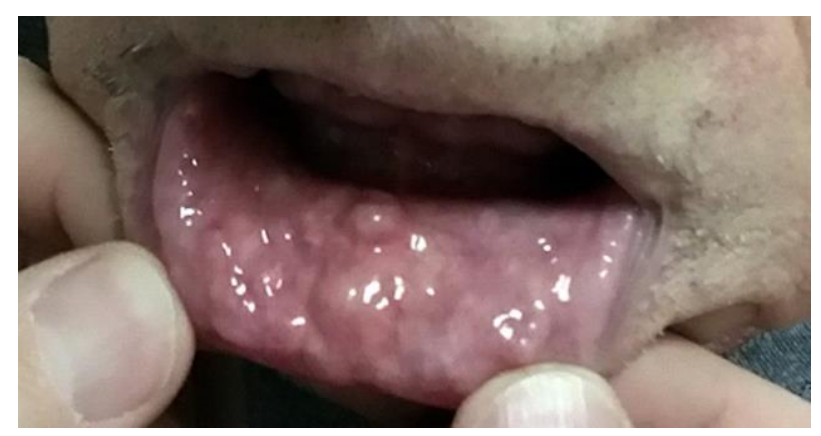

Fig. 1. Hypertrophie des glandes salivaires accessoires.

L'hypertrophie des glandes salivaires accessoires faisait suspecter une amylose AL compliquant un myélome multiple à IgG Kappa. L'analyse anatomopathologique d'une biopsie des glandes salivaires accessoires et d'un segment d'artère temporale révélait un dépôt amyloïde sur les colorations au rouge Congo et en fluorescence jaune-vert en lumière polarisée. Une coloration complémentaire au permanganate, confirmait l'amylose AL. Le bilan d'extension de cette amylose permettait de supposer une atteinte rénale (protéinurie constituée de $80 \%$ d'albumine). Une atteinte osseuse, musculaire était également possible en raison des anomalies de signal à l'IRM de l'épaule. L'atteinte cardiaque n'était pas confirmée sur l'aspect du myocarde en échographie [1]. Si l'atteinte de l'artère temporale est classique dans une artérite à cellules géantes (ACG), l'atteinte de la langue et notamment la macroglossie n'est pas une manifestation écartant cette vascularite [2]. Une protéinurie chez des patients porteurs d'une ACG a pu révéler une amylose secondaire avec atteinte rénale [3] qui a motivé la biopsie de l'artère temporale pour différencier une amylose AL d'une amylose AA. L'anatomopathologie montrait des parois artérielles épaissies par d'importants dépôts d'amylose sans vascularite. Le patient présentait une claudication de la mâchoire par atteinte amyloïdienne du complexe artérielle carotidien externe. Cette symptomatologie bien que rare, a déjà été décrite dans la littérature [4,5]. Au sein d'une cohorte de 237 patients atteints d'amylose AL, $9 \%$ avaient une claudication de la mâchoire. Sur ces 22 patients, 4 avaient un myélome multiple [5]. Après la troisième cure, l'évolution était partiellement favorable avec diminution du galbe des épaules et des muscles fessiers, diminution des douleurs rhizoméliques, de la claudication de la mâchoire et nette régression de l'atteinte neurologique. Il est actuellement toujours en traitement.

\section{Déclaration d'intérêts}

Les auteurs déclarent ne pas avoir de conflits d'intérêts en relation avec cet article. 


\section{Remerciements}

Les auteurs tiennent à remercier le service BUmerang de la B.C.U. de Clermont-Ferrand, son équipe et tout particulièrement Mme Bussemey, ainsi que le service et l'équipe BIUM Info de la B.I.U. Santé, pour leur aide à la recherche documentaire pour cet article.

\section{Références}

[1] Isabel C, Georgin-Lavialle S, Aouba A, Delarue R, Nochy D, Karras A, et al. Amylose cardiaque : à propos d'une série de 14 patients, description et facteurs pronostiques. Rev Med Interne 2013;34:671-8.

[2] Hamidou M, Buzelin F, Rojouan J, Barrier JH, Brisseau JM, Grolleau JY. Syndrome de l'artère temporale au cours d'une amylose AL associée à un myélome. Rev Med Interne 1991;12:306-8.

[3] Monteagudo M, Vidal G, Andreu J, Oristrell J, Tolosa C, Larrosa M, et al. Giant cell (temporal) arteritis and secondary renal amyloidosis: report of 2 cases. J Rheumatol 1997;24:605-7.

[4] Gertz MA, Kyle RA, Griffing WL, Hunder GG. Jaw claudication in primary systemic amyloidosis. Medicine (Baltimore) 1986;65:173-9.

[5] Churchill CH, Abril A, Krishna M, Callman ML, Ginsburg WW. Jaw claudication in primary amyloidosis: unusual presentation of a rare disease. J Rheumatol 2003;30:2283-6. 